\title{
200.
}

\section{ON THE CONES WHICH PASS THROUGH A GIVEN CURVE OF THE THIRD ORDER IN SPACE.}

[From the Philosophical Magazine, vol. xII. (1856), pp. 20-22.]

THE following investigation is connected with the theory of the cubic

$$
\left(a, b, c, d \curlyvee(x, y)^{3}\right. \text {, }
$$

and in particular with a theorem that the determinant formed with the second differential coefficients of the discriminant gives the square of the discriminant.

Consider the coefficients $a, b, c, d$ as linear functions of coordinates, the equations

$$
a c-b^{2}=0, \quad b c-a d=0, \quad b d-c^{2}=0
$$

(equivalent, of course, to two equations) belong to a curve of the third order in space, the edge of regression of the developable surface obtained by putting the discriminant equal to zero, or which has for its equation

$$
-a^{2} d^{2}+6 a b c d-4 a c^{3}-4 b^{3} d+3 b^{2} c^{2}=0 ;
$$

and, moreover, the above forms are the general representations of any curve of the third order, and developable surface of the fourth order. The question arises, to find the equation of the cone of the third order having an arbitrary point for its vertex, and passing through the curve of the third order. This may be done by Joachimsthal's method: let $\alpha, \beta, \gamma, \delta$ be the values of $a, b, c, d$ at the vertex of the cone, and in the equations of the curve, say in

$$
a c-b^{2}=0, \quad b d-c^{2}=0,
$$

for $a, b, c, d$ write $u a+v \alpha, u b+v \beta, u c+v \gamma, u d+v \delta$; if from the equations so obtained $u, v$ are eliminated, the resulting equation will be that of the cone of the third order. 
The substitutions in question give

$$
\begin{aligned}
& L u^{2}+2 M u v+N v^{2}=0, \\
& L^{\prime} u^{2}+2 M^{\prime} u v+N^{\prime} v^{2}=0,
\end{aligned}
$$

where

$$
\begin{array}{lll}
L=2\left(a c-b^{2}\right), & M=a \gamma+c \alpha-2 b \beta, & N=2\left(\alpha \gamma-\beta^{2}\right), \\
L^{\prime}=2\left(b d-c^{2}\right), & M^{\prime}=b \delta+d \beta-2 c \gamma, & N^{\prime}=2\left(\beta \delta-\gamma^{2}\right)
\end{array}
$$

the result of the elimination is

$$
4\left(L N-M^{2}\right)\left(L^{\prime} N^{\prime}-M^{\prime 2}\right)-\left(L N^{\prime}+L^{\prime} N-2 M M^{\prime}\right)^{2}=0,
$$

and we have

$$
\begin{aligned}
L N-M^{2} & =4(b \gamma-c \beta)(a \beta-b \alpha)-(c \alpha-a \gamma)^{2}, \\
L^{\prime} N^{\prime}-M^{\prime 2} & =4(c \delta-d \gamma)(b \gamma-c \beta)-(b \delta-d \gamma)^{2}, \\
L N^{\prime}+L^{\prime} N-2 M M^{\prime} & =4(b \gamma-c \beta)^{2}+4(a \beta-b \alpha)(c \delta-d \gamma)+2(c \alpha-a \gamma)(b \delta-d \beta) .
\end{aligned}
$$

Write for shortness

$$
\begin{aligned}
& b \gamma-c \beta=l, \quad c \alpha-a \gamma=m, \quad a \beta-b \alpha=n, \\
& a \delta-d \alpha=f, \quad b \delta-d \beta=g, \quad c \delta-d \gamma=h,
\end{aligned}
$$

values which give $l f+m g+n h=0$. Then forming the expression

$$
4\left(4 l n-m^{2}\right)\left(4 h l-g^{2}\right)-\left(4 l^{2}+4 n h+2 m g\right)^{2},
$$

this is equal to $\left({ }^{1}\right)$

$$
-16 l\left(l^{3}-n f h+l m g-2 l n h+n g^{2}+m^{2} h\right)
$$

and the equation of the cone is

$$
l^{3}-n f h+l m g-2 l n h+\bar{n} g^{2}+m^{2} h=0 ;
$$

or, substituting and expanding,

$$
\begin{aligned}
&\left(\alpha \delta^{2}-\gamma^{3}\right) b^{3}-\left(\alpha^{2} \delta-\beta^{3}\right) c^{3}-\delta\left(\beta \delta-\gamma^{2}\right) a b^{2}+\alpha\left(\alpha \gamma-\beta^{2}\right) d c^{2} \\
&+ \delta\left(\beta \delta-\gamma^{2}\right) a^{2} c-\alpha\left(\alpha \gamma-\beta^{2}\right) d^{2} b+2 \delta\left(\alpha \gamma-\beta^{2}\right) a c^{2}-2 \alpha\left(\beta \delta-\gamma^{2}\right) d b^{2} \\
&-\gamma\left(\beta \delta-\gamma^{2}\right) a^{2} d+\beta\left(\alpha \gamma-\beta^{2}\right) a d^{2}+3 \gamma(\beta \gamma-\alpha \delta) b^{2} c-3 \beta(\beta \gamma-\alpha \delta) b c^{2} \\
&+ \delta(\beta \gamma-\alpha \delta) a b c-\alpha(\beta \gamma-\alpha \delta) d b c+\left(\alpha \gamma \delta-3 \beta \gamma^{2}+2 \beta^{2} \delta\right) a b d \\
&-\left(\alpha \beta \delta-3 \beta^{2} \gamma+2 \alpha \gamma^{2}\right) a c d=0 .
\end{aligned}
$$

1 With respect to the occurrence of the factor $l(=b \gamma-c \beta)$, it is worth noticing, that, putting $b=k \beta$, $c=k \gamma$, we have identically

$$
\begin{aligned}
& L u^{2}+2 M u v+N v^{2}=2\left[(a \gamma-b \beta) u+\left(a \gamma-\beta^{2}\right) v\right](k u+v), \\
& L^{\prime} u^{2}+2 M^{\prime} u v+N^{\prime} v^{2}=2\left[(\beta \delta-c \gamma) u+\left(\beta \delta-\gamma^{2}\right) v\right](k u+v),
\end{aligned}
$$

i.e. the two functions will contain a common factor if $b=k \beta, c=k \gamma$, or what is the same thing, if $b \gamma-c \beta=0$. But if the functions contain a common factor, their resultant vanishes, i.e. the resultant will vanish in virtue of the relation $b \gamma-c \beta=0$, or what is the same thing, $b \gamma-c \beta$ is a factor of the resultant. 
Now putting

$$
b d-c^{2}=p, \quad b c-a d=q, \quad a c-b^{2}=r,
$$

and in like manner

$$
\beta \delta-\gamma^{2}=P, \quad \beta \gamma-\alpha \delta=Q, \quad \alpha \gamma-\beta^{2}=R,
$$

and reducing, the final result may be expressed in the form

$$
\begin{aligned}
& P\{-2 \alpha b p+(\gamma a-2 \beta b) q+(\delta a-\gamma c) r\} \\
+ & Q\{-\alpha c p+(\gamma b-\beta c) q+\quad \delta b r\} \\
+ & R\{-(\alpha d-\beta c) p-(\beta d-2 \gamma c) q+\quad 2 \delta c r\}=0,
\end{aligned}
$$

where $a, b, c, d$ are current coordinates, and $p, q, r$ are quadratic functions of $a, b, c, d$. The equation is (as it should be) satisfied by the equations $(p=0, q=0, r=0)$ of the given curve; it is also satisfied per se when $P=0, Q=0, R=0$, i.e. when the vertex is a point on the curve; this indicates a change in form of the equation, and in fact the cone is in this case of the second order only. Suppose that the coordinates of the vertex are in this case given by $\frac{\alpha}{\beta}=\frac{\beta}{\gamma}=\frac{\gamma}{\delta}=\frac{1}{\sigma}$ ( $\sigma$ an arbitrary quantity), it may be easily shown that the equation of the cone is

$$
p+\sigma q+\sigma^{2} r=0
$$

or at full length

$$
\left(b d-c^{2}\right)+\sigma(b c-a d)+\sigma^{2}\left(a c-b^{2}\right)=0 ;
$$

in fact this equation is evidently that of a surface of the second order passing through the curve; and there is no difficulty in showing that it is a cone.

2, Stone Buildings, May 1, 1856. 